IP Periodica Polytechnica Civil Engineering

59(3), pp. 423432,2015

DOI: $10.3311 /$ PPci.7819

Creative Commons Attribution (1)

RESEARCH ARTICLE

\section{Fracture of a Circular Disk with Mixed Conditions on the Boundary}

Vagif M. Mirsalimov, Nailya M. Kalantarly

Received 15-11-2014, revised 10-03-2015, accepted 26-03-2015

\begin{abstract}
A model of a circular disk fracture based on consideration of the fracture process zone near the curvilinear crack tip is suggested. It is considered that mixed boundary conditions are given on the boundary of the disk. It is accepted that the fracture process zone is a finite length layer with a material with partially broken bonds between its separate structural elements (end zone). Analysis of equilibrium limit of the curvilinear crack is performed on the basis of ultimate extension of the material bonds.
\end{abstract}

\section{Keywords}

circular disk · mixed boundary conditions $\cdot$ curvilinear crack · crack with interfacial bonds $\cdot$ cohesive forces

\section{Vagif M. Mirsalimov}

Institute of Mathematics and Mechanics of NAS of Azerbaijan, AZ-1141 Baku, B.Vahabzade, 9, Azerbaijan

e-mail: vagif.mirsalimov@imm.az

\section{Nailya M. Kalantarly}

Institute of Mathematics and Mechanics of NAS of Azerbaijan, AZ-1141 Baku, B.Vahabzade 9, Azerbaijan

e-mail: nailyak1975@gmail.com

\section{Introduction}

Circular disks are widely used in up-to-date machines. The problems of disks strength are very urgent and undoubtedly, interest to these problems will increase in connection with development of machine-building and power engineering. For analyzing the disks reliability it is necessary to study their stressstrain state and fracture. Simulation and analysis of stress-strain state in disks has a special applied value in the first turn for tame choice of their construction, optimal sizes and admissible value of actuating loadings. The disks often work in highly stressful conditions. There is a wide reference (see review in the monographs [1,2]) devoted to strength analysis of disks. In most existing papers A. Griffith's model of a crack is used.

Account of plastic deformation near the crack was realized by M.Ya. Leonov and V.V. Panasyuk [3] and afterwards by D.S. Dugdale [4]. Bilby and others [5,6] obtained the solution of the problem at longitudinal shear. By analogy of D.S. Dugdale's hypothesis, it was accepted that the plastic zone was concentrated in the narrow layer on the continuation of the crack.

As applied to the problem of brittle failure, G.I. Barenblatt has suggested the conception of cohesion zone. Implicit existence of bonds between stresses near the crack ends and its continued faces was supposed in [7].

The model of a crack with interfacial bonds at the end zones may be used in different scales of fracture. Intensive development of crack models with explicit account of nonlinear laws of interaction in conformity to elasto-visco-plastic behaviour of materials and various kinds of loading is connected with this fact. Bibliography of works on this theme may be found in the papers of special issue [8].

In the present paper we use a bridged crack's model [8-10].

\section{Formulation of the problem}

Assume that on the boundary of a circular disk the normal displacements $u_{r}(t)$ and tangential component of surface forces $N_{\theta}(t)$ are given, and the normal pressure $N_{r}(t)$ should be defined in the course of problem solution. Refer the cross section of the disk to polar system of coordinates $r \theta$ having chosen the origin of coordinates at the centre of the circle $L$ of radius $R$ (Fig. 1 ). 
Let the disk be weakened by a crack. In real materials, because of structural and technological factors the crack surfaces have irregularities and curvatures. In the disk's cross section, the crack with end zones is represented by a slot of length $2 \ell=b-a$, whose contour has small deviations from the rectilinear form (Fig. 1).

The crack is assumed to be close to the rectilinear form allowing only small deviations of the crack line from the straight line $y=0$.

The crack contour equation is accepted in the form

$$
y=f(x), \quad a \leq x \leq b .
$$

Based on the accepted assumption on the form of the crack with end zones, the functions $f(x)$ and $f^{\prime}(x)$ are small quantities relative to the crack length. Let us consider some arbitrary realization of the curved surface of the crack.

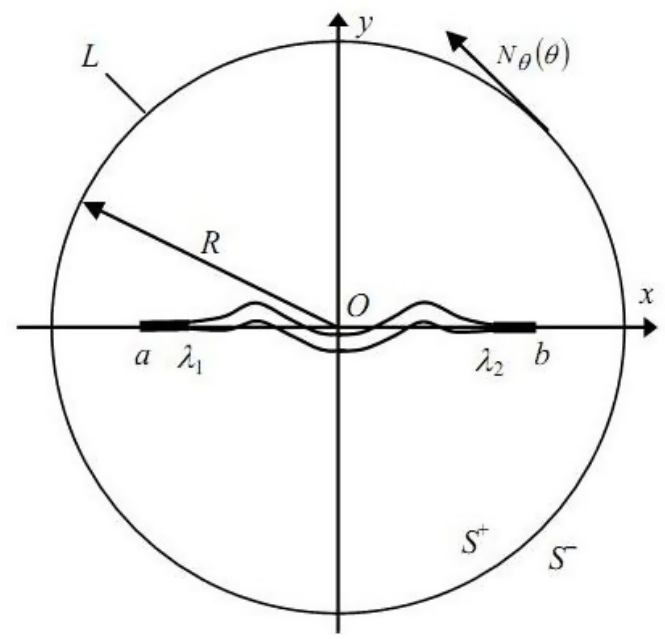

Fig. 1. Computatinal diagram of fracture mechanics problem for a circular disk

As the disk is loaded, in the crack vertices there will arise the prefracture zones (end zones) that we model as the areas of weakened interparticle bonds of the material. Interaction of faces of these areas is simulated by introducing between the prefracture zone faces the bonds having the given deformation diagram. Physical nature of such bonds and the sizes of prefracture zones where the interaction of faces of weakened interparticle bonds is realized, depends on the kind of the material. The bonds between the crack faces at the end zones retard the fracture development. This braking effect grows by increasing the size of the end zone of the crack occupied by the bonds [11-13]. In the case when the size of the crack end zone is not small in comparison with the crack length, the approximate methods of estimation of fracture toughness of disks based on consideration of a small end zone crack, are not applicable. In these cases direct simulation of stress state at the end zone of the crack with regard to deformation characteristics of bonds is necessary.

Distinguish the parts of the crack $d_{1}=\lambda_{1}-a$ and $d_{2}=$ $b-\lambda_{2}$ (end areas), where the crack faces interact. Interaction of the crack faces at the end zones is simulated by introducing be- tween the crack faces the bonds (cohesive forces) with the given deformation diagram. As the circular disk is loaded, in the bonds connecting the crack faces there will arise normal $q_{y}(x)$ and tangential $q_{x y}(x)$ forces. These stresses are not known beforehand and should be defined.

The boundary conditions on the faces of the crack with end zones are of the form

$$
\begin{aligned}
& \sigma_{n}-i \tau_{n t}=0 \text { for } y=f(x), \lambda_{1}<x<\lambda_{2} \\
& \sigma_{n}-i \tau_{n t}=q_{y}(x)-i q_{x y}(x) \text { for } y=f(x), \\
& a \leq x \leq \lambda_{1} \text { and } \lambda_{2} \leq x \leq b .
\end{aligned}
$$

The main relations of the problem should be complemented by the equation connecting the opening of end zone faces and forces in bonds. Without loss of generality, represent this equation in the form [9]

$$
\begin{aligned}
& \left(v^{+}-v^{-}\right)-i\left(u^{+}-u^{-}\right)=\Pi(x, \sigma) \cdot \\
& \cdot\left[q_{y}(x)-i q_{x y}(x)\right] \\
& \sigma=\sqrt{q_{y}^{2}+q_{x y}^{2}}
\end{aligned}
$$

The function $\Pi(x, \sigma)$ is the effective compliance of the bonds dependent on tension; $\sigma$ is a modulus of a stress vector in bonds; $\left(v^{+}-v^{-}\right)$is a normal, $\left(u^{+}-u^{-}\right)$is a tangential component of the opening of end zone faces of the crack.

Denote the considered area enclosed between the circle $L$ of radius $R$ and one slit $L_{1}=[a, b]$ by $S^{+}$, the area complemented to the complete complex plane by $S^{-}$.

Since the functions $f(x)$ and $f^{\prime}(x)$ are small quantities, we can represent the function $f(x)$ in the form

$$
f(x)=\varepsilon H(x), a \leq x \leq b,
$$

where $\varepsilon$ is a small parameter.

\section{The Method of the Boundary-Value Problem Solution}

Using the perturbations method, we get boundary conditions for $y=0, a \leq x \leq b$ : for a zero approximation

$$
\begin{aligned}
& \sigma_{y}^{(0)}-i \tau_{x y}^{(0)}=0 \text { for } y=0, \lambda_{1}^{0}<x<\lambda_{2}^{0} \\
& \sigma_{y}^{(0)}-i \tau_{x y}^{(0)}=q_{y}^{(0)}-i q_{x y}^{(0)} \text { for } y=0 \\
& a \leq x \leq \lambda_{1}^{0} \text { and } \lambda_{2}^{0} \leq x \leq b
\end{aligned}
$$

for a first approximation

$$
\begin{aligned}
& \sigma_{y}^{(1)}-i \tau_{x y}^{(1)}=\bar{q}_{y}-i \bar{q}_{x y} \text { for } y=0, \lambda_{1}^{1}<x<\lambda_{2}^{1}, \\
& \sigma_{y}^{(1)}-i \tau_{x y}^{(1)}=q_{y}^{(1)}-i q_{x y}^{(1)}+\bar{q}_{y}-i \bar{q}_{x y} \text { for } y=0, \\
& a \leq x \leq \lambda_{1}^{1} \text { and } \lambda_{2}^{1} \leq x \leq b .
\end{aligned}
$$


Here

$$
\begin{aligned}
& q_{y}(x)=q_{y}^{(0)}(x)+\varepsilon q_{y}^{(1)}(x) ; \\
& q_{x y}(x)=q_{x y}^{(0)}(x)+\varepsilon q_{x y}^{(1)}(x) ; \\
& \lambda_{1}=\lambda_{1}^{0}+\varepsilon \lambda_{1}^{1} ; \lambda_{2}=\lambda_{2}^{0}+\varepsilon \lambda_{2}^{1} ; \\
& \bar{q}_{y}=2 \tau_{x y}^{(0)} \frac{d H}{d x}-H \frac{\partial \sigma_{y}^{(0)}}{\partial y} \text { for } y=0 ; \\
& \bar{q}_{x y}=\left(\sigma_{y}^{(0)}-\sigma_{x}^{(0)}\right) \frac{d H}{d x}-H \frac{\partial \tau_{x y}^{(0)}}{\partial y} .
\end{aligned}
$$

Similarly, we get boundary conditions on the contour $L$ at each approximation, and also the relations connecting the opening of the end zone faces of the crack and forces in bonds.

Now construct the solution in a zero approximation. Denote the area occupied by a disk, bounded by a circle $L$ and one rectilinear cut $[a, b]$ by $S^{+}$, the area is complemented to complete complex plane by $S^{-}$.

Under these conjectures, the problem is reduced to definition of two complex variable functions $\Phi_{0}(z)$ and $\Psi_{0}(z)$, analytic in the area $S^{+}$and satisfying on the basis of [14] the following boundary conditions:

$$
\begin{aligned}
& \operatorname{Re}\left\{\kappa \Phi_{0}(t)-\overline{\Phi_{0}(t)}+\frac{R^{2}}{t^{2}}\left[t \overline{\Phi_{0}^{\prime}(t)}+\overline{\Psi_{0}(t)}\right]\right\}= \\
& =2 \mu u_{r}^{\prime}(t)
\end{aligned}
$$

on $L$,

$$
\begin{aligned}
& \operatorname{Im}\left\{\Phi_{0}(t)+\overline{\Phi_{0}(t)}-\frac{t^{2}}{R^{2}}\left[\bar{t} \Phi_{0}^{\prime}(t)+\Psi_{0}(t)\right]\right\}= \\
& =-N_{\theta}(t)
\end{aligned}
$$

on $L$.

And also the condition on the faces of the crack with end zones

$$
\Phi_{0}(x)+\overline{\Phi_{0}(x)}+x \overline{\Phi_{0}^{\prime}(x)}+\overline{\Psi_{0}(x)}=f_{0}(x),
$$

where $\kappa=(3-v) /(1+v) ; v$ is the Poisson ratio of the disk's material; $\mu$ is the shear modulus;

$$
f_{0}(x)=\left\{\begin{array}{llr}
0 & y=0, & \lambda_{1}^{0}<x<\lambda_{2}^{0} \\
q_{y}^{(0)}(x)-i q_{x y}^{(0)}(x) & y=0, & a \leq x \leq \lambda_{1}^{0} \\
& & \lambda_{2}^{0} \leq x \leq b
\end{array}\right.
$$

In the general case, on the circle $L$ we take the functions $u_{r}(t)$ and $N_{\theta}(t)$ in the form of Fourier series:

$$
u_{r}(t)=\sum_{v=-\infty}^{\infty} V_{v}\left(\frac{t}{R}\right)^{v}, \quad i N_{\theta}(t)=\sum_{v=-\infty}^{\infty} T_{v}\left(\frac{t}{R}\right)^{v},
$$

where $V_{v}, T_{v}(v=0, \pm 1, \pm 2, \ldots)$ are generally speaking, known complex coefficients and are determined by the formulas

$$
\begin{aligned}
& V_{k}=\frac{1}{2 \pi} \int_{0}^{2 \pi} u_{r}(\theta) e^{i k \theta} d \theta \quad k=0,1,2, \ldots \\
& T_{k}=\frac{1}{2 \pi} \int_{0}^{2 \pi} i N_{\theta}(\theta) e^{i k \theta} d \theta .
\end{aligned}
$$

The main relations of the stated problem in a zero approximation should be complemented by the equation connecting the opening of the faces of the prefracture end zone and the forces in bonds.

$$
\begin{aligned}
& v_{0}^{+}(x, 0)-v_{0}^{-}(x, 0)-i\left(u_{0}^{+}(x, 0)-u_{0}^{-}(x, 0)\right)= \\
& =\Pi\left(x, \sigma^{0}\right)\left(q_{y}^{(0)}(x)-i q_{x y}^{(0)}(x)\right)
\end{aligned}
$$

Passing in relations (8) and (9) to conjugate values, after some transformations on the contour $L$ we get boundary conditions in the following form

$$
\begin{aligned}
& (\kappa-1)\left[\Phi_{0}(t)+\overline{\Phi_{0}(t)}\right]+\frac{R^{2}}{t^{2}}\left[t \overline{\Phi_{0}^{\prime}(t)}+\overline{\Psi_{0}(t)}\right]+ \\
& +\frac{t^{2}}{R^{2}}\left[\frac{R^{2}}{t} \Phi_{0}^{\prime}(t)+\Psi_{0}(t)\right]=4 \mu u_{r}^{\prime}(t)
\end{aligned}
$$

on $L$.

$$
\begin{aligned}
& -\frac{R^{2}}{t^{2}}\left[t \overline{\Phi_{0}^{\prime}(t)}+\overline{\Psi_{0}(t)}\right]+\frac{t^{2}}{R^{2}}\left[\frac{R^{2}}{t} \Phi_{0}^{\prime}(t)+\Psi_{0}(t)\right]= \\
& =2 i N_{\theta}(t)
\end{aligned}
$$

on $L$

Based on (15) and (16), on the circle $L$ we shall have the following relation

$$
\begin{aligned}
& (\kappa-1)\left[\Phi_{0}(t)+\overline{\Phi_{0}(t)}\right]+\frac{2 t^{2}}{R^{2}}\left[\frac{R^{2}}{t} \Phi_{0}^{\prime}(t)+\Psi_{0}(t)\right]= \\
& =2\left[2 \mu u_{r}^{\prime}(t)+i N_{\theta}(t)\right]
\end{aligned}
$$

on $L$.

Now, substitute relation (12) into the last equality and get:

$$
\begin{aligned}
& (\kappa-1)\left[\Phi_{0}(t)+\overline{\Phi_{0}(t)}\right]+2 \frac{t^{2}}{R^{2}}\left[\frac{R^{2}}{t} \Phi_{0}^{\prime}(t)+\Psi_{0}(t)\right]= \\
& =2\left\{\sum_{v=0}^{\infty}\left[T_{v}+\frac{2 \mu(v+1)}{R} V_{v+1}\right]\left(\frac{t}{R}\right)^{v}+\right. \\
& \left.+\sum_{v=1}^{\infty}\left[T_{-v}-\frac{2 \mu(v-1)}{R} V_{-v+1}\right]\left(\frac{R}{t}\right)^{v}\right\}
\end{aligned}
$$

on $L$.

Introduce on $L$ a new unknown auxiliary function $\omega_{0}(t) \in H$ (the Holder condition) in the form

$$
\begin{aligned}
& 2 \omega_{0}(t)=(\kappa-1)\left[\Phi_{0}(t)-\overline{\Phi_{0}(t)}\right]- \\
& -\frac{2 t^{2}}{R^{2}}\left[\frac{R^{2}}{t} \Phi_{0}^{\prime}(t)+\Psi_{0}(t)\right] \text { on L. }
\end{aligned}
$$

Putting together relations (18) and (19), we get

$$
\begin{aligned}
& \Phi_{0}(t)=\frac{\omega_{0}(t)}{\kappa-1}+\frac{1}{\kappa-1} \sum_{v=0}^{\infty}\left[\frac{2 \mu(v+1)}{R} V_{v+1}+T_{v}\right]\left(\frac{t}{R}\right)^{v}+ \\
& +\frac{1}{\kappa-1} \sum_{v=1}^{\infty}\left[T_{-v}-\frac{2 \mu(v-1)}{R} V_{-v+1}\right]\left(\frac{R}{t}\right)^{v} \text { on L. }
\end{aligned}
$$

Now, having substituted (20) into (19), we get

$$
\Psi(t)=Q(t)+R_{1}(t)+R_{2}(t) \text { on } \mathrm{L} .
$$


Here we introduce the following denotation

$$
\begin{aligned}
& R_{1}(t)=\sum_{v=0}^{\infty}\left[\frac{1}{2}\left(1-\frac{v-2}{\kappa-1}\right) T_{v+2}-\frac{1}{2} \bar{T}_{-v-2}+\right. \\
& \left.+\frac{\mu(v+1)}{R} V_{-v-1}+\frac{\mu(v+3)}{R}\left(1-\frac{v-2}{\kappa-1}\right) V_{v+3}\right]\left(\frac{t}{R}\right)^{v} ; \\
& R_{2}(t)=-\sum_{v=2}^{\infty}\left[\frac{\mu(v-1)}{R} \bar{V}_{v-1}+\frac{1}{2} \bar{T}_{v-2}\right]\left(\frac{R}{t}\right)^{v}+ \\
& \sum_{v=3}^{\infty}\left[\frac{1}{2}\left(1+\frac{v-2}{\kappa-1}\right) T_{-v+2}-\frac{\mu(v-3)}{R}\left(1+\frac{v-2}{\kappa-1}\right) V_{-v+3}\right] \times \\
& \times\left(\frac{R}{t}\right)^{v}++\left[\frac{\mu}{R} V_{1}+\frac{1}{2} T_{0}\right] \frac{R^{2}}{t^{2}}+ \\
& +\left[\frac{1}{2}\left(1-\frac{1}{\kappa-1}\right) T_{1}-\frac{1}{2} \bar{T}_{-1}-\frac{\mu}{(\kappa-1) R} V_{1}+\frac{2 \mu}{R} V_{2}\right] \frac{R}{t} \\
& Q(t)=-\frac{R^{2}}{2 t^{2}}\left[\omega_{0}(t)+\overline{\omega_{0}(t)}\right]-\frac{R^{2}}{(\kappa-1) t} \omega_{0}^{\prime}(t) .
\end{aligned}
$$

Based on the theorem on analytic continuation, and the properties of the Cauchy type integral, from relations 20 and 21 we get:

$$
\begin{aligned}
& \Phi_{*}(z)=\Phi_{0}(z)-\frac{1}{\kappa-1} \frac{1}{2 \pi i} \int_{L} \frac{\omega_{0}(t)}{t-z} d t- \\
& -\frac{1}{\kappa-1} \sum_{v=0}^{\infty}\left[\frac{2 \mu(v+1)}{R} V_{v+1}+T_{v}\right]\left(\frac{z}{R}\right)^{v} \quad z \in S^{+} ; \\
& \Phi_{*}(z)=-\frac{1}{\kappa-1} \frac{1}{2 \pi i} \int_{L} \frac{\omega_{0}(t) d t}{t-z}+ \\
& +\frac{1}{\kappa-1} \sum_{v=1}^{\infty}\left[T_{-v}-\frac{2 \mu(v-1)}{R} V_{-v+1}\right]\left(\frac{R}{z}\right)^{v} \quad z \in S^{-} ; \\
& \Psi_{*}(z)=\left\{\begin{array}{ll}
\Psi_{0}(z)-\frac{1}{2 \pi i} \int_{L} \frac{Q(t)}{t-z} d t-R_{1}(z), & z \in S^{+} \\
-\frac{1}{2 \pi i} \int_{L} \frac{Q(t)}{t-z} d t+R_{2}(z), & z \in S^{-}
\end{array} .\right.
\end{aligned}
$$

In relations 23$]-25]$ the functions $\Phi_{*}(z)$ and $\Psi_{*}(z)$ are analytic in the complete complex plane cut along the slit $L=[a, b]$ and vanish at infinity, i.e.

$$
\Phi_{*}(\infty)=0 ; \quad \Psi_{*}(\infty)=0 .
$$

We shall look for the unknown auxiliary function $\omega_{0}(t) \in H$ on $L$ in the form of Fourier series

$$
\omega_{0}(t)=\alpha_{0}+\sum_{v=1}^{\infty}\left[\alpha_{v}\left(\frac{t}{R}\right)^{v}+\alpha_{-v}\left(\frac{R}{t}\right)^{v}\right],
$$

where $\alpha_{v}(v=0, \pm 1, \pm 2, \ldots)$ are, generally speaking, complex coefficients.

We substitute relation (27) in the first formulas of (23), 25] and using the Cauchy integral theorem get general formulas for the sought-for functions:

$$
\Phi_{0}(z)=\Phi_{*}(z)+\sum_{v=0}^{\infty} J_{v}\left(\frac{z}{R}\right)^{v} z \in S^{+},
$$

$$
\Psi_{0}(z)=\Psi_{*}(z)+\sum_{v=0}^{\infty} W_{v}\left(\frac{z}{R}\right)^{v} z \in S^{+}
$$

Here

$$
\begin{aligned}
& J_{v}=\frac{1}{\kappa-1}\left[\alpha_{v}+T_{v}+\frac{2 \mu(v+1)}{R} V_{v+1}\right] \\
& W_{v}=-\left(\frac{1}{2}+\frac{v+2}{\kappa-1}\right) \alpha_{v+2}-\frac{1}{2} \bar{\alpha}_{-v-2}+\frac{1}{2}\left(1-\frac{v+2}{\kappa-1}\right) T_{v+2}- \\
& -\frac{1}{2} \bar{T}_{-v-2}+\frac{\mu(v+3)}{R}\left(1-\frac{v+2}{\kappa-1}\right) V_{v+3}+\frac{\mu(v+1)}{R} \bar{V}_{-v-1}
\end{aligned}
$$

For determining the functions $\Phi_{*}(z)$ and $\Psi_{*}(z)$, following [14] we consider the function

$$
\Omega_{*}(z)=\bar{\Phi}_{*}(z)+z{\overline{\Phi^{\prime}}}_{*}(z)+\bar{\Psi}_{*}(z)
$$

that is analytic on the whole complex plane outside of the rectilinear slit (i.e. the cracks with end zones).

For the stress vector components we have [9]:

$$
\sigma_{y}^{(0)}-i \tau_{x y}^{(0)}=\Phi_{0}(z)+\overline{\Phi_{0}(z)}+z \overline{\Phi_{0}(z)}+\overline{\Psi_{0}(z)} .
$$

Taking into account the loading condition on the faces of crack and on the end zones, based on (10) as $z \rightarrow t(t$ is the affix of the points of crack and end zones) we get the conditions

$$
\begin{aligned}
& \Phi_{0}^{+}(t)+\bar{\Phi}_{0}^{-}(t)+t \bar{\Phi}_{0}^{-^{\prime}}(t)+\bar{\Psi}_{0}^{-}(t)=f_{0} ; \\
& \Phi_{0}^{-}(t)+\bar{\Phi}_{0}^{+}(t)+t \bar{\Phi}_{0}^{+^{\prime}}(t)+\bar{\Psi}_{0}^{+}(t)=f_{0},
\end{aligned}
$$

where $f_{0}=0$ on the crack faces and $f_{0}=q_{y}^{(0)}-i q_{x y}^{(0)}$ in the end zones.

Substituting formulas (28), 29) in relation (33), we have

$$
\begin{aligned}
& \Phi_{*}^{+}(t)+\bar{\Phi}_{*}^{-}(t)+t \overline{\Phi^{\prime}}{ }_{0}^{-}(t)+\bar{\Psi}_{*}^{-}(t)=f_{1}(t)+f_{0} ; \\
& \Phi_{*}^{-}(t)+\bar{\Phi}_{*}^{+}(t)+t{\overline{\Phi^{\prime}}}_{0}^{+}(t)+\bar{\Psi}_{*}^{+}(t)=f_{1}(t)+f_{0},
\end{aligned}
$$

where

$$
f_{1}(t)=-\sum_{v=0}^{\infty}\left[J_{v}+(v+1) \bar{J}_{v}+\bar{W}_{v}\right]\left(\frac{t}{R}\right)^{v}
$$

Having changed in formula 31 by $\bar{z}$ and passing to conjugated values, we get

$$
\Psi_{*}(z)=\bar{\Omega}_{*}(z)-\Phi_{*}(z)-z \Phi_{*}^{\prime}(z)
$$

Having substituted (38) in relation (35), we get

$$
\Phi_{*}^{+}(t)+\Omega_{*}^{-}(t)=f_{*}(t)
$$

$$
\Phi_{*}^{-}(t)+\Omega_{*}^{+}(t)=f_{*}(t) .
$$


Hence we obtain that the problem of definition of the functions $\Phi_{*}(z)$ and $\Omega_{*}(z)$ is reduced to the Riemann linear conjugation problem [14]:

$$
\begin{gathered}
{\left[\Phi_{*}(t)+\Omega_{*}(t)\right]^{+}+\left[\Phi_{*}(t)+\Omega_{*}(t)\right]^{-}=f_{*}(t) ;} \\
{\left[\Phi_{*}(t)-\Omega_{*}(t)\right]^{+}-\left[\Phi_{*}(t)-\Omega_{*}(t)\right]^{-}=0 .}
\end{gathered}
$$

Here

$$
\begin{aligned}
& f_{*}(t)=2 f_{0}+2 f_{1}(t)=\sum_{v=0}^{\infty}\left(\ell_{v}+p_{v}\right)\left(\frac{t}{R}\right)^{v}+2 f_{0} ; \\
& \ell_{v}=-2\left[-\left(\frac{1}{2}+\frac{v+2}{\kappa-1}\right) \bar{\alpha}_{v+2}-\frac{1}{2} \alpha_{-v-2}+\frac{1}{\kappa-1} \alpha_{v}+\right. \\
& \left.+\frac{v+1}{\kappa-1} \bar{\alpha}_{v}\right] ; \\
& p_{v}=-2\left[\frac{1}{2}\left(1-\frac{v+2}{\kappa-1}\right) \bar{T}_{v+2}-\frac{1}{2} T_{-v-2}+\frac{1}{\kappa-1} T_{v}+\right. \\
& +\frac{v+1}{k_{0}-1} \bar{T}_{v}+\frac{2 \mu(v+1)}{(\kappa-1) R} V_{v+1}+\frac{2 \mu(v+1)^{2}}{(\kappa-1) R} \bar{V}_{v+1}+ \\
& \left.+\frac{\mu(v+1)}{R} V_{-v-1}+\frac{\mu(v+3)}{R}\left(1-\frac{v+2}{\kappa-1}\right) \bar{V}_{v+3}\right] .
\end{aligned}
$$

Since $\Phi_{*}(\infty)-\Omega_{*}(\infty)=0$, then the general solution of problem (42) will be

$$
\Phi_{*}(z)-\Omega_{*}(z)=0
$$

Based on (41) and (44), for the function $\Phi_{*}(z)$ we get a linear conjugation problem

$$
\begin{gathered}
\Phi_{*}^{+}(x)+\Phi_{*}^{-}(x)=f_{1}(x) \quad \lambda_{1}^{0}<x<\lambda_{2}^{0}, \\
P h i_{*}^{+}(x)+\Phi_{*}^{-}(x)=q_{y}^{(0)}(x)-i q_{x y}^{(0)}(x)+f_{1}(x) \\
a \leq x \leq \lambda_{1}^{0} \text { and } \lambda_{2}^{0} \leq x \leq b .
\end{gathered}
$$

The corresponding homogeneous problem is of the form

$$
\Phi_{*}^{+}(x)+\Phi_{*}^{-}(x)=0 \quad a \leq x \leq b .
$$

Since the stresses in the disk are restricted, the solution of the boundary value problem (45) should be sought in the class of everywhere bounded functions.

As a particular solution of homogeneous problem (47) we take the function

$$
X(z)=\sqrt{(z-a)(z-b)}
$$

meaning the branch for which the following equality holds

$$
X^{+}(x)=-X^{-}(x) \text { on } a \leq x \leq b .
$$

Based on the last relation, we rewrite the conjugation problem 47) as follows

$$
\frac{\Phi_{*}^{+}(x)}{X^{+}(x)}-\frac{\Phi_{*}^{-}(x)}{X^{-}(x)}=0 \text { on } a \leq x \leq b
$$

From the last boundary condition it follows that the solution of the homogeneous problem vanishing at infinity equals zero.

We represent the inhomogeneous conjugation problem 45 in the form

$$
\frac{\Phi_{*}^{+}(x)}{X^{+}(x)}-\frac{\Phi_{*}^{-}(x)}{X^{-}(x)}=\frac{F(x)}{X^{+}(x)} \text { on } a \leq x \leq b
$$

Denote

$$
\Phi_{0 *}(z)=\frac{\Phi_{*}(z)}{X^{+}(z)} ; F_{*}(x)=\frac{F(x)}{X^{+}(x)},
$$

then boundary value problem 51) takes the form

$$
\begin{aligned}
& \Phi_{0 *}^{+}(z)-\Phi_{0 *}^{-}(z)=F_{*}(x) \text { on } a<x<b . \\
& \text { Here } F_{*}(x)=\frac{f_{1}(x)}{\sqrt{(x-b)(x-a)}} \text { for } \lambda_{1}^{0}<x<\lambda_{2}^{0}, \\
& F_{*}(x)=\frac{q_{y}^{(0)}-i q_{x y}^{(0)}+f_{1}(x)}{\sqrt{(x-b)(x-a)}} \\
& \text { for } a \leq x \leq \lambda_{1}^{0} \text { and } \lambda_{2}^{0} \leq x \leq b .
\end{aligned}
$$

The desired solution of the problem is written as [9]:

$$
\Phi_{*}(z)=\frac{\sqrt{(z-b)(z-a)}}{2 \pi i} \int_{a}^{b} \frac{F_{*}(x) d x}{x-z} .
$$

According to the behavior of the function $\Phi_{*}(z)$ at infinity, the solvability condition of the boundary value problem has the form

$$
\int_{a}^{b} \frac{f_{*}(t) d t}{\sqrt{(t-a)(b-t)}}=0 ; \quad \int_{a}^{b} \frac{t f_{*}(t) d t}{\sqrt{(t-a)(b-t)}}=0
$$

These relations help to find the unknown parameters $\lambda_{1}^{0}$ and $\lambda_{2}^{0}$ determining the sizes of the end zones of the crack at a zero approximation.

The obtained relation contains the unknown stresses at the end zones of the crack.

Now we construct an integral equation for determining unknown forces $q_{y}^{(0)}(x)-i q_{x y}^{(0)}(x)$. The additional relation (14) is the condition that determines the unknown stresses in the bonds between the faces at the end zones of the crack in a zero approximation.

In the considered problem it is convenient to write this additional condition for the derivative of the opening of the faces of the crack's end zones.

Using the Kolosov-Muskhelishvili relation [14] and boundary values of the functions $\Phi_{*}(z)$ and $\Omega_{*}(z)$,on the segment $y=0$, $a \leq x \leq b$ we get the following equality

$$
\begin{aligned}
& \Phi_{0}^{+}(x)-\Phi_{0}^{-}(x)= \\
& =\frac{2 \mu}{1+\kappa}\left[\frac{\partial}{\partial x}\left(u_{0}^{+}-u_{0}^{-}\right)+i \frac{\partial}{\partial x}\left(v_{0}^{+}-v_{0}^{-}\right)\right] .
\end{aligned}
$$

Using the Sokhotskii-Plemelj formulas [14] and taking into account formula (54), we find

$$
\begin{aligned}
& \Phi_{0}^{+}(x)-\Phi_{0}^{-}(x)=-\frac{i \sqrt{(x-a)(x-b)}}{\pi} \times \\
& \times\left[\int_{a}^{b} \frac{f_{*}(t) d t}{\sqrt{(t-a)(t-b)}(t-x)}\right] .
\end{aligned}
$$


We substitute the found expression into the left hand side of (56), and taking into account relation (14) after some transformations we find the system of nonlinear integro-differential equations with respect to the unknown functions $q_{y}^{(0)}$ and $q_{x y}^{(0)}$ :

$$
\begin{aligned}
& -\frac{1}{\pi} \sqrt{(x-a)(b-x) \times} \\
& \times\left[\int_{a}^{b} \frac{q_{y}^{(0)}(t) d t}{\sqrt{(t-a)(b-t)}(t-x)}+\right. \\
& \left.+\int_{a}^{b} \frac{f_{y}(t) d t}{\sqrt{(t-a)(b-t)}(t-x)}\right]= \\
& =\frac{2 \mu}{1+\kappa} \frac{d}{d x}\left(\Pi\left(x, \sigma^{0}\right) q_{y}^{(0)}(x)\right) \\
& -\frac{1}{\pi} \sqrt{(x-a)(b-x) \times} \\
& \times\left[\int_{a}^{b} \frac{q_{x y}^{(0)}(t) d t}{\sqrt{(t-a)(b-t)}(t-x)}+\right. \\
& \left.+\int_{a}^{b} \frac{2 \mu}{\sqrt{(t-a)(b-t)}(t-x)}\right]= \\
& =\frac{2 \mu}{1+\kappa} \frac{d}{d x}\left(\Pi\left(x, \sigma^{0}\right) q_{x y}^{(0)}(x)\right)
\end{aligned}
$$

Here $f_{y}(t)=\operatorname{Re} f_{1}(t) ; f_{x y}(t)=\operatorname{Im} f_{1}(t) ;$

$$
f_{1}(t)=-\sum_{v=0}^{\infty}\left[J_{v}+(v+1) \bar{J}_{v}+\bar{W}_{v}\right]\left(\frac{t}{R}\right)^{v}
$$

Each of the equations 58 and (59) is a non-linear integrodifferential equation with the Cauchy kernel and may be solved only numerically. For solving them we can use the collocational scheme with approximation of unknown functions [15]. The obtained relations 28) - 29) with regard to formulas 20, (21), 27) and equations (58), 59 permit to get the final solution of the problem in a zero approximation if the coefficients $\alpha_{v}(v=0, \pm 1, \pm 2, \ldots)$ are determined.

For composing the infinite system of algebraic equations with respect to unknowns $\alpha_{k}$, substitute relations (28), 29) into condition (18) with regard to (54) and expansions

$$
\begin{aligned}
& \sqrt{(t-a)(t-b)}=t \sum_{r=0}^{\infty} M_{r}\left(\frac{R}{t}\right)^{r}, \\
& \frac{1}{\sqrt{(t-a)(t-b)}}=\sum_{r=0}^{\infty} M_{r}^{*}\left(\frac{R}{t}\right)^{r+1}
\end{aligned}
$$

After some transformations, condition $(18)$ is reduced to the form

$$
\begin{aligned}
& \sum_{m=0}^{\infty} A_{m}\left(\frac{t}{R}\right)^{m}+\sum_{m=0}^{\infty} A_{m}^{*}\left(\frac{R}{t}\right)^{m}= \\
& =\sum_{m=0}^{\infty} C_{m}\left(\frac{t}{R}\right)^{m}+\sum_{m=0}^{\infty} C_{m}^{*}\left(\frac{R}{t}\right)^{m} .
\end{aligned}
$$

In view of some bulky form of expressions for $A_{m}, A_{m}^{*}, C_{m}$, $C_{m}^{*}(m=0,1,2, \ldots)$ they are not cited. Comparing the coefficients at the same degrees $t / R$ and $R / t$ in the both sides of the obtained relation (62), we get infinite systems of linear algebraic equations

$$
\begin{aligned}
& A_{0}+A_{0}^{*}=C_{0}+C_{0}^{*} \quad(m=0) \\
& A_{m}=C_{m}, \quad A_{m}^{*}=C_{m}^{*} \quad(m=1,2, \ldots) .
\end{aligned}
$$

Now let us pass to procedure for converting a system to an algebraic system of integro-differential equations (58) and 59) with additional conditions (55). At first in integro-differential equations (58) and (59) and in additional conditions (55) all integration intervals are reduced to one interval $[-1,1]$. By means of quadrature formulas all integrals are replaced by finite sums, and the derivatives in the right sides of equations 58 and (59) are replaced by finite-difference approximations. Therewith the following boundary conditions are taken into account: $q_{y}(a)=q_{y}(b)=0 ; q_{x y}(a)=q_{x y}(b)=0$ (this corresponds to the conditions $v_{0}^{+}(a, 0)-v_{0}^{-}(a, 0)=0 ; v_{0}^{+}(b, 0)-v_{0}^{-}(b, 0)=0$; $\left.u_{0}^{+}(a, 0)-u_{0}^{-}(a, 0)=0 ; u_{0}^{+}(b, 0)-u_{0}^{-}(b, 0)=0\right)$. As a result, instead of each integral equation with corresponding additional conditions, we get $M_{1}+2$ algebraic equations for determining the stresses at the nodal points contained at the end zone of the crack, and the sizes of the end zones.

$$
\begin{aligned}
& \sum_{k=1}^{M_{1}} A_{m k}\left(q_{y, k}^{(0)}+f_{y, k}\right)=\frac{1+\kappa}{4 \mu} \frac{M}{b-a} \times \\
& \times\left[\Pi\left(x_{m+1}, \sigma^{0}\left(x_{m+1}\right)\right) q_{y, m+1}^{(0)}-\Pi\left(x_{m-1}, \sigma^{0}\left(x_{m-1}\right)\right) q_{y, m-1}^{(0)}\right] \\
& \left(m=1,2, \ldots, M_{1}\right), \\
& \sum_{k=1}^{M} f_{* y}\left(\cos \theta_{k}\right)=0, \quad \sum_{k=1}^{M} \tau_{k} f_{* y}\left(\tau_{k}\right)=0 .
\end{aligned}
$$

$$
\begin{aligned}
& \sum_{k=1}^{M_{1}} A_{m k}\left(q_{x y, k}^{(0)}+f_{x y, k}\right)=\frac{1+\kappa}{4 \mu} \frac{M}{b-a} \times \\
& \times\left[\Pi\left(x_{m+1}, \sigma^{0}\left(x_{m+1}\right)\right) q_{x y, m+1}^{(0)}-\Pi\left(x_{m-1}, \sigma^{0}\left(x_{m-1}\right)\right) q_{x y, m-1}^{(0)}\right]
\end{aligned}
$$$$
\left(m=1,2, \ldots, M_{1}\right) \text {, }
$$

$$
\begin{aligned}
& \sum_{k=1}^{M} f_{* x y}\left(\tau_{k}\right)=0, \\
& \sum_{k=1}^{M} \tau_{k} f_{* x y}\left(\tau_{k}\right)=0 . \\
& \text { Here } q_{y, k}^{(0)}=q_{y}^{(0)}\left(\tau_{k}\right), q_{x y, k}^{(0)}=q_{x y}^{(0)}\left(\tau_{k}\right), \\
& f_{y, k}=f_{y}\left(\tau_{k}\right), f_{x y, k}=f_{x y}\left(\tau_{k}\right), \\
& x_{m+1}=\frac{a+b}{2}+\frac{b-a}{2} \eta_{m+1}, A_{m k}=-\frac{1}{M} \cot \frac{\theta_{m} \mp \theta_{k}}{2} .
\end{aligned}
$$

The joint solution of the obtained equations permits at the given characteristics of bonds to determine the forces in the 
bonds $q_{y}^{(0)}(x), q_{x y}^{(0)}(x)$ and the sizes of the end zones (parameters $\lambda_{1}^{0}$ and $\lambda_{2}^{0}$ ) at a zero approximation.

After solving the obtained algebraic systems, we can pass to construction of basic resolving equations of the problem in a first approximation. According to the found solution we find the functions $\bar{q}_{y}(x)$ and $\bar{q}_{x y}(x)$.

The succession of the solutions of the problem in a first approximation is similar to a zero approximation. In a first approximation the problem is reduced to determination of two analytic functions $\Phi_{1}(z)$ and $\Psi_{1}(z)$, analytic in the domain $S^{+}$and satisfying the following boundary conditions

$$
\begin{gathered}
\operatorname{Re}\left\{\kappa \Phi_{1}(t)-\overline{\Phi_{1}(t)}+\frac{R^{2}}{t^{2}}\left[t \overline{\Phi_{1}^{\prime}(t)}+\overline{\Psi_{1}(t)}\right]\right\}=0 \text { on } L, \\
\operatorname{Im}\left\{\Phi_{1}(t)+\overline{\Phi_{1}(t)}-\frac{t^{2}}{R^{2}}\left[\bar{t} \Phi_{1}^{\prime}(t)+\Psi_{1}(t)\right]\right\}=0 \text { on } L, \\
\Phi_{1}(x)+\overline{\Phi_{1}(x)}+x \overline{\Phi_{1}^{\prime}(x)}+\overline{\Psi_{1}(x)}=f_{1}(x)
\end{gathered}
$$

$$
(a \leq x \leq b),
$$

where

$$
f_{1}(x)=\left\{\begin{array}{lll}
\bar{q}_{y}-i \bar{q}_{x y} y=0, & y=0, & \lambda_{1}^{1}<x<\lambda_{2}^{1} \\
q_{x y}^{(1)}-i q_{y}^{(1)}+\bar{q}_{y}-i \bar{q}_{x y} & y=0, & a \leq x \leq \lambda_{1}^{1} \\
& \lambda_{2}^{1} \leq x \leq b
\end{array}\right.
$$

Repeating the above mentioned method for solving the boundary value problem in a zero approximation, we find

$$
\omega_{1}(t)=\alpha_{0}^{1}+\sum_{v=1}^{\infty}\left[\alpha_{v}^{1}\left(\frac{t}{R}\right)^{v}+\alpha_{-v}^{1}\left(\frac{R}{t}\right)^{v}\right],
$$

$$
\begin{aligned}
& \Phi_{1}(z)=\Phi_{*}(z)+\sum_{v=0}^{\infty} J_{v}^{1}\left(\frac{z}{R}\right)^{v} \quad z \in S^{+}, \\
& \Psi_{1}(z)=\Psi_{*}(z)+\sum_{v=0}^{\infty} W_{v}^{1}\left(\frac{z}{R}\right)^{v} \quad z \in S^{+},
\end{aligned}
$$

Here $J_{v}^{1}=\frac{\alpha_{v}^{1}}{\kappa-1} ; W_{v}^{1}=-\left(\frac{1}{2}+\frac{v+2}{\kappa-1}\right) \alpha_{v+2}^{1}-\frac{1}{2} \bar{\alpha}_{-v-2}$.

The complex potentials have the form

$$
\Omega_{*}(z)=\Phi_{*}(z)=\frac{\sqrt{(z-a)(z-b)}}{2 \pi i} \int_{a}^{b} \frac{F_{*}^{1}(x) d x}{x-z},
$$

Here

$$
\begin{aligned}
& F_{*}^{1}(x)=\frac{f_{1}^{1}(x)+\bar{q}_{y}-i \bar{q}_{x y}}{\sqrt{(x-a)(x-b)}} \text { for } \lambda_{1}^{1}<x<\lambda_{2}^{1} ; \\
& F_{*}^{1}(x)=\frac{q_{y}^{(1)}-i q_{x y}^{(1)}+\bar{q}_{y}-i \bar{q}_{x y}+f_{1}^{1}(x)}{\sqrt{(x-a)(x-b)}}
\end{aligned}
$$

for $a \leq x \leq \lambda_{1}^{1}$ and $\lambda_{2}^{1} \leq x \leq b$;

$$
\begin{aligned}
& f_{1}^{1}=\sum_{v=0}^{\infty} \ell_{v}^{1}\left(\frac{t}{R}\right)^{v} ; \\
& \ell_{v}^{1}=-2\left[-\left(\frac{1}{2}+\frac{v+2}{\kappa-1}\right) \bar{\alpha}_{v+2}^{1}-\frac{1}{2} \alpha_{-v-2}+\frac{1}{\kappa-1} \alpha_{v}^{1}+\right. \\
& \left.+\frac{v+1}{\kappa-1} \bar{\alpha}_{v}^{1}\right] .
\end{aligned}
$$

The solvability conditions of the boundary value problem in a first approximation have the form

$$
\int_{a}^{b} F_{*}^{1}(t) d t=0 ; \quad \int_{a}^{b} t F_{*}^{1}(t) d t=0 .
$$

These equations are used to determine the unknown parameters $\lambda_{1}^{1}$ and $\lambda_{2}^{1}$ defining the sizes of the crack's end zones in a first approximation.

The obtained relations contain unknown stresses at the crack's end zones.

Now construct integral equations for finding the unknown forces $q_{y}^{(1)}(x)-i q_{x y}^{(1)}(x)$. The additional relation

$$
\begin{aligned}
& v_{1}^{+}(x, 0)-v_{1}^{-}(x, 0)-i\left(u_{1}^{+}(x, 0)-u_{1}^{-}(x, 0)\right)= \\
& =\Pi\left(x, \sigma^{1}\right)\left(q_{y}^{(1)}(x)-i q_{x y}^{(1)}(x)\right)
\end{aligned}
$$

is the condition that determines the unknown stresses in the bonds between the faces in the crack's end zones in a first approximation.

Behaving as in a zero approximation, after some transformations we find the system of nonlinear integro-differential equations with respect to the unknown functions $q_{y}^{(1)}$ and $q_{x y}^{(1)}$ :

$$
\begin{aligned}
& -\frac{1}{\pi} X(x)\left[\int_{a}^{b} \frac{q_{y}^{(1)}(t) d t}{X(x)(t-x)}+\int_{a}^{b} \frac{f_{y}^{1}(t) d t}{X(x)(t-x)}\right]= \\
& \frac{2 \mu}{1+\kappa} \frac{d}{d x}\left(\Pi\left(x, \sigma^{1}\right) q_{y}^{(1)}(x)\right) ; \\
& -\frac{1}{\pi} X(x)\left[\int_{a}^{b} \frac{q_{x y}^{(1)}(t) d t}{X(x)(t-x)}+\int_{a}^{b} \frac{f_{x y}^{1}(t) d t}{X(x)(t-x)}\right]= \\
& \frac{2 \mu}{1+\kappa} \frac{d}{d x}\left(\Pi\left(x, \sigma^{1}\right) q_{x y}^{(1)}(x)\right) ;
\end{aligned}
$$

Here $f_{y}^{1}(t)=\operatorname{Re} f_{1}^{1}(t)+\bar{q}_{y}(t)$

$$
\begin{aligned}
& f_{x y}^{1}(t)=\bar{q}_{x y}+\operatorname{Im} f_{1}^{1}(t) \\
& f_{1}^{1}(t)=-\sum_{v=0}^{\infty}\left[J_{v}^{1}+(v+1) \bar{J}_{v}^{1}+\bar{W}_{v}^{1}\right]\left(\frac{t}{R}\right)^{v} .
\end{aligned}
$$


The obtained relations (71) - 75, allowing for formulas

$$
\begin{aligned}
& \Phi_{1}(t)=\frac{\omega_{1}(t)}{\kappa-1} \\
& \Psi_{1}(t)=-\frac{R^{2}}{2 t^{2}}\left[\omega_{1}(t)+\overline{\omega_{1}(t)}\right]-\frac{R^{2}}{(\kappa-1) t} \omega_{1}^{\prime}(t)
\end{aligned}
$$

and equations (77), 78) permit to obtain the final solution of the problem in a first approximation if the coefficients $\alpha_{v}^{1}(v=0, \pm 1, \pm 2, \ldots)$ are determined.

For constructing the infinite system of linear algebraic equations for the coefficients $\alpha_{v}^{1}$ of the auxiliary function $\omega_{1}(t)$, we behave as in a zero approximation. As a result, we get two infinite systems of linear algebraic equations of type 63 .

As a result of procedure for converting integro-differential equations (77) and (78) with additional conditions (75), instead of each integral equation with corresponding additional conditions, to an algebraic system we get $M_{1}+2$ algebraic equations for determining the stresses at the nodal points contained at the crack's end zones, and the sizes of the end zones in a first approximation:

$$
\begin{aligned}
& \sum_{k=1}^{M_{1}} A_{m k}\left(q_{y, k}^{(1)}+f_{y, k}^{1}\right)=\frac{1+\kappa}{4 \mu} \frac{M}{b-a} \times \\
& \times\left[\Pi\left(x_{m+1}, \sigma^{1}\left(x_{m+1}\right)\right) q_{y, m+1}^{(1)}-\Pi\left(x_{m-1}, \sigma^{1}\left(x_{m-1}\right)\right) q_{y, m-1}^{(1)}\right]
\end{aligned}
$$

$$
\begin{gathered}
\left(m=1,2, \ldots, M_{1}\right), \\
\sum_{k=1}^{M} f_{y}^{1}\left(\cos \theta_{k}\right)=0, \\
\sum_{k=1}^{M} \tau_{k} f_{y}^{1}\left(\tau_{k}\right)=0 . \\
\sum_{k=1}^{M_{1}} A_{m k}\left(q_{x y, k}^{(1)}+f_{x y, k}^{1}\right)=\frac{1+\kappa}{4 \mu} \frac{M}{b-a} \times \\
\times\left[\Pi\left(x_{m+1}, \sigma^{1}\left(x_{m+1}\right)\right) q_{x y, m+1}^{(1)}-\right. \\
\left.-\Pi\left(x_{m-1}, \sigma^{1}\left(x_{m-1}\right)\right) q_{x y, m-1}^{(1)}\right]\left(m=1,2, \ldots, M_{1}\right), \\
\sum_{k=1}^{M} f_{x y}^{1}\left(\tau_{k}\right)=0, \sum_{k=1}^{M} \tau_{k} f_{x y}^{1}\left(\tau_{k}\right)=0 .
\end{gathered}
$$

Here

$$
\begin{aligned}
& q_{y, k}^{(1)}=q_{y}^{(1)}\left(\tau_{k}\right), q_{x y, k}^{(1)}=q_{x y}^{(1)}\left(\tau_{k}\right), \\
& f_{y, k}^{1}=f_{y}^{1}\left(\tau_{k}\right), f_{x y, k}^{1}=f_{x y}^{1}\left(\tau_{k}\right) .
\end{aligned}
$$

The remaining denotations are the same as in a zero approximation.

The joint solution of the obtained equations permits at the given characteristics of bonds to determine the forces in the bonds $q_{y}^{(1}(x)$ and $q_{x y}^{(1)}(x)$, the sizes of the end zones (the parameters $\lambda_{1}^{1}$ and $\lambda_{2}^{1}$ ) and also to study the influence of irregularities and curvatures of the crack surface on the stress strain state of the circular disk.

\section{Numerical Solution and Analysis}

For formulation of the limit equilibrium criterion, we use the criterion of critical opening of the crack. The opening of the crack in the range of end zones may be determined from the relations

$$
\begin{aligned}
& v^{+}(x, 0)-v^{-}(x, 0)=\Pi(x, \sigma) q_{y}(x), \\
& a \leq x \leq \lambda_{1} \text { and } \lambda_{2} \leq x \leq b, \\
& u^{+}(x, 0)-u^{-}(x, 0)=\Pi(x, \sigma) q_{x y}(x) .
\end{aligned}
$$

The condition of critical opening of the crack near the edge of the end zone will be

$$
\begin{aligned}
& \Pi\left(\lambda_{1}, \sigma\left(\lambda_{1}\right)\right) \sigma\left(\lambda_{1}\right)=\delta_{c} \text { for } x=\lambda_{1}, \\
& \Pi\left(\lambda_{2}, \sigma\left(\lambda_{2}\right)\right) \sigma\left(\lambda_{2}\right)=\delta_{c} \text { for } x=\lambda_{2},
\end{aligned}
$$

where $\delta_{c}$ is the characteristics of the disk material determined experimentally.

Even in the special case of linear-elastic bonds, the obtained systems of equations become nonlinear because of unknown sizes of the crack's end zones. In this connection for solving the obtained systems in the case of linear bonds, the successive approximations method was used. At each approximation the algebraic system was solved numerically by the Gauss method with choice of the main element. In the case of nonlinear law of deformation of bonds, for determining the forces at the end zones, an algorithm similar to the A.A. Il'yushin method of elastic solutions [16] was also used. Analysis of effective compliance is carried out similar to definition of the secant modulus in the method of variables of elasticity parameters [17]. The successive approximations process ends as soon as the forces along the end zone, obtained at two successive iterations differ a little from each other.

The nonlinear part of the curve of deformation of the bonds was taken in the form of bilinear dependence whose ascending portion corresponded to elastic deformation of bonds $\left(0<V(x)<V_{*}, V=\sqrt{u^{2}+v^{2}}\right)$ with maximum tension of bonds. For $V(x)>V_{*}$ the deformation law was described by a nonlinear relation which is determined by two points $\left(V_{*}, \sigma_{*}\right)$, and $\left(\delta_{c}, \sigma_{c}\right)$, and for $\sigma_{c} \geq \sigma_{*}$ we have increasing linear dependence (linear strengthening corresponding to elasto-plastic deformation of the bonds). The algebraic system with respect to tractions in bonds was solved numerically. For numerical calculations it was assumed $M=30$ that responds to partition of integration interval into 30 Chebyshev nodal points.

The plots of dependence of dimensionless length of the end zones of the right end $d_{2}=\left(b-\lambda_{2}\right) /\left(b-\lambda_{2}\right)(b-a)$ on dimensionless load $N_{0} / \sigma_{*}$ for different sizes of cracks $\ell_{*}=$ $\left(\lambda_{2}-\lambda_{1}\right) /(b-q, a)$ are depicted in Fig. 2

Fig. 3 represents the graphs of distribution of the normal tractions $q_{y} / N_{0}$ in the bonds for the right end zone of the crack (curve 1 for linear bonds, curve 2 for bilinear curve of deformation of bonds).

The dependence of dimensionless opening modulus $\delta_{*}=$ 
$\pi E \delta / 8 N_{0} \ell$ for the right end of the crack on dimensionless parameter $N_{0} / \sigma_{*}$ is represented in Fig. 4 .

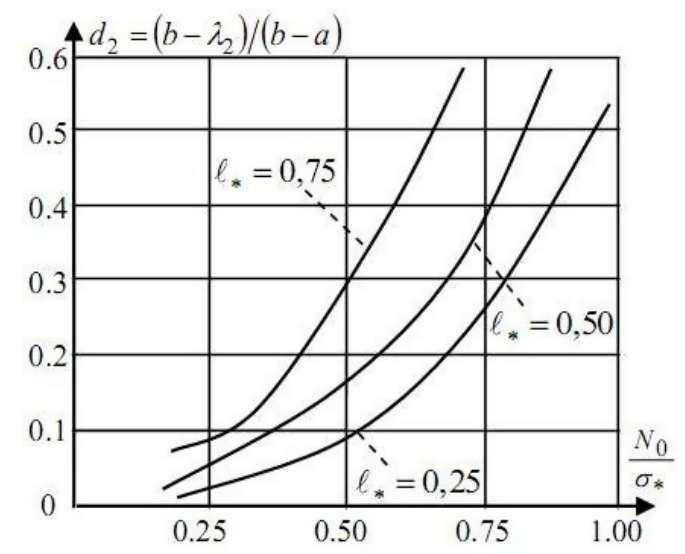

Fig. 2. Dependence of dimensionless length of the end zones of the right end $d_{2}=\left(b-\lambda_{2}\right) /(b-a)$ on dimensionless load $N_{0} / \sigma_{*}$ for different sizes of cracks $\ell_{*}=\left(\lambda_{2}-\lambda_{1}\right) /(b-a)$

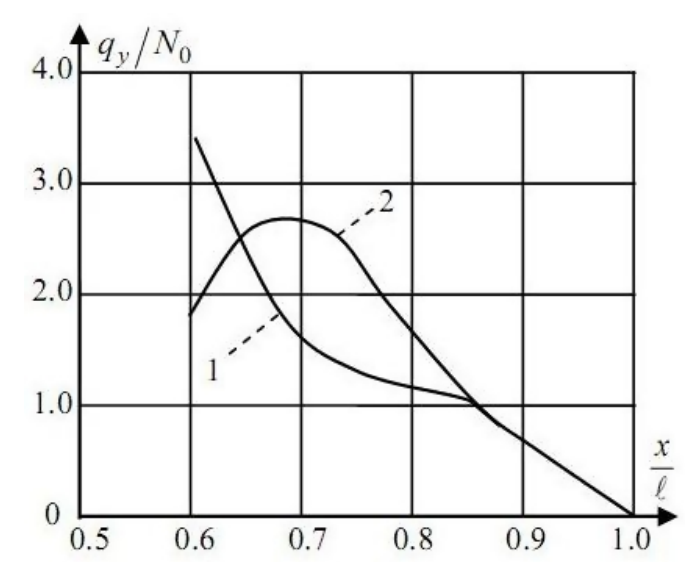

Fig. 3. Distribution of normal forces $q_{y} / N_{0}$ in the bonds for the right end zone of the crack (curve 1 corresponds to linear law of deformation of bonds, curve 2 to nonlinear deformation)

The obtained main resolving algebraic equations permit to predict the ultimate admissible size of the crack (technological defect) by means of numerical calculation for a specific circular disk.

\section{Conclusions}

The obtained closed algebraic system of equations and limit condition of the crack growth permits to determine the admissible size of the crack for different laws of deformation of interparticle bonds, elastic and geometrical characteristics of the material and the circular disk by means of numerical calculation for any specific circular disk.

Distribution of forces in bonds and opening of crack faces is determined directly from the solution of the obtained algebraic systems. The model of a bridged crack at the end zones enables to study main regularities of forces distributions at the end zones, to make analysis of ultimate equilibrium state of the curved crack by means of deformation criterion.

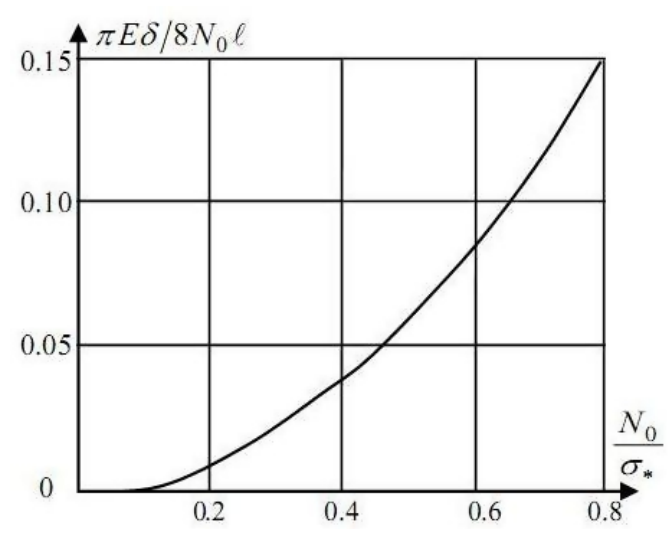

Fig. 4. Dependence of dimensionless opening modulus $\delta_{*}=\pi E \delta / 8 N_{0} \ell$ for the right end of the crack on dimensionless parameter $N_{0} / \sigma_{*}$

The developed calculation method permits to solve the following practically important problems:

to estimate the guaranteed resource of the disk with regard to expected deficiency and loading conditions;

to set admissible level of deficiency and maximum value of actuating loads that provide sufficient safety margin;

to select the disk's material with a complex of characteristics of fracture toughness.

The model of a crack with end zones, permits to consider the fracture process including crack initiation, crack formation and crack development from unified positions.

\section{References}

1 Savruk MP, Fracture mechanics and strength of materials. Vol. 2. Stress intensity factors in cracked bodies, Naukova dumka; Kiev, 1988.

2 Savruk MP, Osiv PN, Prokopchuk IV, Numerical analysis in plane problems of cracks theory, Naukova dumka; Kiev, 1989.

3 Leonov MY, Panasyuk VV, Развитие мельчайших трещин в твердом теле" (Development of smallest cracks in the solid), International Applied Mechanics, 5(4), (1959), 391-401. (In Russian).

4 Dugdale DS, Yielding of steel sheets containing slits, J. Mech. and Phys. Solids, 8(2), (1960), 100-108, DOI 10.1016/0022-5096(60)90013-2

5 Bilby BA, Cottrell AH, Smith E, Swinden $\mathbf{K H}$, Plastic yielding from sharp notches, Proc. R. Soc. Lond. A, 279(1376), (1964), 1-9, DOI 10.1098/rspa.1964.0085

6 Field FA, Yielding in a cracked plate under longitudinal shear, J. Appl. Mech, 30(4), (1963), 622-623, DOI 10.1115/1.3636629

7 Barenblatt GI, Математическая теория равновесных трещин, образующихся при хрупком разрушении" (The mathematical theory for equilibrium cracks formed on brittle fracture), J. of Applied Mechanics and Technical Physics, 2(4), (1961), 3-56. (In Russian).

8 The special issue: Cohesive models, Eng. Fract. Mech., 70(14), (2003), 1741-1987, DOI 10.1016/S0013-7944(03)00121-8

9 Mirsalimov VM, The solution of a problem in contact fracture mechanics of the nucleation and development of a bridged crack in the hub of a friction pair, J. of Applied Math. and Mech., 71(1), (2007), 120-136, DOI 10.1016/j.jappmathmech.2007.03.003

10 Mirsalimov VM, Hasanov FF, Interaction between Periodic System of Rigid Inclusions and Rectilinear Cohesive Cracks in an Isotropic Medium under Transverse Shear, Acta Polytechnica Hungarica, 11(5), (2014), 161-176, DOI 10.12700/APH.11.05.2014.05.10

11 Rose LRF, Crack reinforcement by distributed springs, J. Mech. Phys. Solids, 35(4), (1987), 383-405, DOI 10.1016/0022-5096(87)90044-5 
12 Cox BN, Marshall DB, Concepts for bridged cracks fracture and fatigue, Acta metal mater., 42(2), (1994), 341-363, DOI 10.1016/09567151(94)90492-8

13 Budianscky B, Cui YL, On the tensile strength of a fiber-reinforced ceramic composite containing a crack like flaw, J. Mechanics and Physics of Solids., 42(1), (1994), 1-19, DOI 10.1016/0022-5096(94)90048-5

14 Muskhelishvili NI, Some Basic Problems of Mathematical Theory of Elasticity, Kluwer; Amsterdam, 1977.

15 Ladopoulos EG, Singular integral equations, linear and non-linear theory and its applications in science and engineering, Springer Verlag; New York, Berlin, 2000.

16 II'yushin AA, Plasticity, Gostexhizdat; Moscow and Leningrad, 1948.

17 Birger IA, Расчет конструкций с учетом пластичности и ползучести" (The design of structures allowings for plasticity and creep), Izv. Akad. Nauk SSSR, Mekhanika, 2, (1965), 113-119. (In Russian). 